\title{
VARIABILITÉ DE L'ENNEIGEMENT ET VARIATIONS DU CLIMAT
}

\author{
Eric Martin, Eric Brun et Yves Durand \\ Météo-France, CNRM \\ Centre d'études de la neige \\ 1441, rue de la piscine \\ 38406 Saint-Martin d'Hères Cedex
}

RÉSUMÉ

L'étude de la climatologie nivale des Alpes françaises, réalisée à partir de séries de mesures sur certains sites, montre que la variabilité spatiale et temporelle de l'enneigement est très grande. En particulier, les conditions de très faible enneigement rencontrées durant certains hivers récents peuvent être comparées à celles de certains hivers du passé.

La modélisation du manteau neigeux est un moyen efficace pour compléter notre connaissance de la climatologie nivale car elle permet de prendre en compte les relations complexes entre conditions météorologiques et enneigement. Elle a également été utilisée pour simuler l'impact d'un réchauffement du climat sur le manteau neigeux. Les résultats montrent la très grande sensibilité de l'enneigement, ce qui conduirait probablement à des modifications importantes pour l'hydrologie, l'écologie et l'économie de ces régions.

The study of the current snow climatology in the French Alps, with series of snow records at selected sites, shows that the spatial and temporal variability of snow cover is very high. In particular, the very poor snow conditions encountered during some recent winters can be compared with those of some winters in the past.

The numerical snow modelling is also a good mean to improve our knowledge because it is able to take into account the complex relations between meteorological conditions and snow. Lastly, it was used to simulate the impact of a climate warming on the alpine snow cover. The results show its high sensitivity, which may induce important perturbations in various domains such as hydrology, ecology and economy in these regions.

Depuis quelques années, à la veille des vacances scolaires d'hiver, les médias nous présentent systématiquement le bilan de santé d'une grande malade : la neige. Il est vrai que certains hivers récents $(88-89,89-90,92-93)$ ont été caractérisés en début de saison par des enneigements faibles, voire inexistants sur les principaux massifs montagneux français. Les conséquences socio-économiques de ces situations ont été telles que beaucoup se sont interrogés sur les liens qui pourraient exister entre ces anomalies répétées et les prémices d'un changement du climat.

Mais la neige ne conditionne pas seulement l'afflux de touristes dans les stations de sports d'hiver. Une modification des conditions d'enneigement peut avoir des conséquences hydrologiques importantes, à court et à long terme. En effet, après un hiver peu enneigé, les débits des rivières sont faibles, avec des problèmes à craindre quant à l'alimentation de certaines nappes phréatiques (et le touriste, déjà peu satisfait par ses vacances d'hiver, éprouvera moins de plaisir à se baigner l'été suivant dans des retenues à moitié vides !). Un réchauffement durable peut également entraîner une disparition progressive des glaciers. Les écosystèmes des régions 


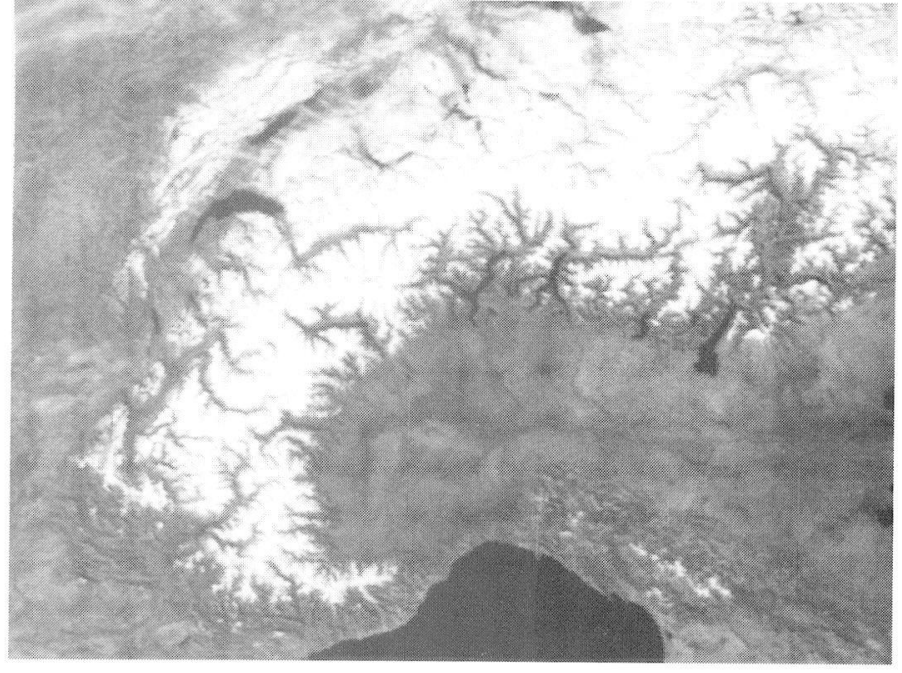

Figure 1 - L'arc alpin enneigé vu par le satellite défilant NOAA (altitude : $850 \mathrm{~km}$ )

montagneuses dépendent, eux aussi, fortement des caractéristiques de l'enneigement. Par exemple, à haute altitude, des variations assez faibles de la durée de la période pendant laquelle le sol est recouvert de neige sont susceptibles d'entraîner des variations importantes de la courte période de croissance des plantes.

Pour savoir si les conditions rencontrées ces derniers hivers sont réellement exceptionnelles, il est tout d'abord nécessaire de connaître les principales caractéristiques de l'enneigement, en particulier sa variabilité naturelle dans l'espace et dans le temps et les phénomènes physiques qui régissent son évolution et son interaction avec l'atmosphère. A partir de cette connaissance, qui a bénéficié des progrès récents en modélisation du manteau neigeux, il est possible de formuler des hypothèses et d'évaluer leurs conséquences sur l'enneigement. Dans la suite, on se limitera au cas des Alpes, pour lesquelles on a le plus d'éléments.

\section{L'ENNEIGEMENT DANS LE PASSÉ}

L'évaluation du caractère exceptionnel du faible enneigement d'un hiver ne peut être menée que par comparaison avec les observations du passé. Mais les mesures de hauteur de neige n'ont été effectuées régulièrement en France que depuis une quarantaine d'années. EDF exploite un réseau de mesures depuis les années 1945 pour l'évaluation des réserves hydro-électriques, et ce n'est que depuis 1959 que Météo-France a introduit systématiquement la mesure de hauteur de neige au sol dans son réseau climatologique de montagne. Plus récemment, le réseau nivométéorologique, constitué de postes situés dans les stations de sports d'hiver, a permis de compléter le réseau de mesures.

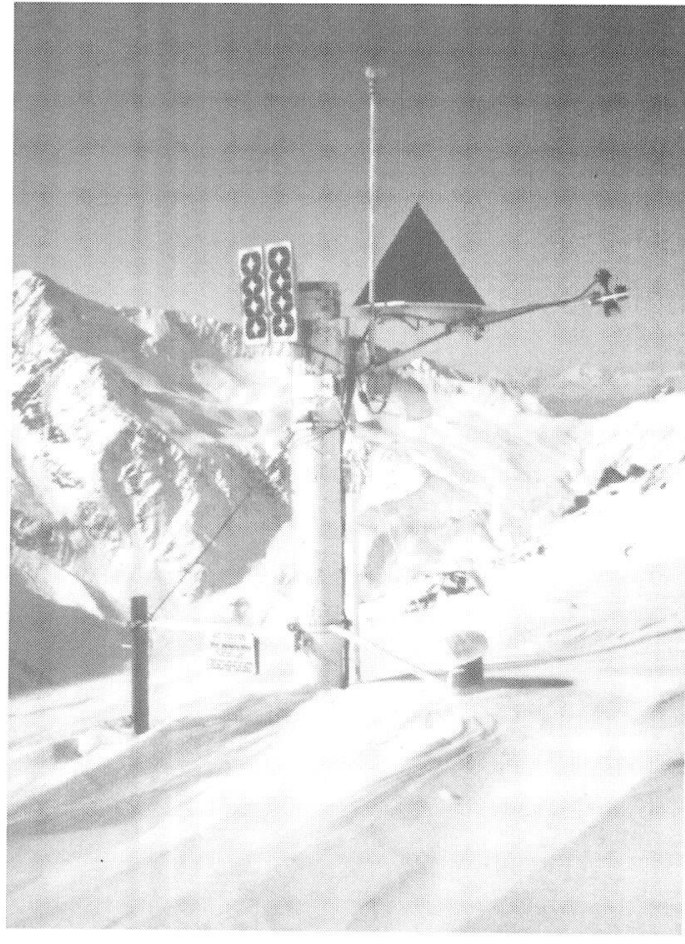

Figure 2 - Station automatique "Nivôse» (massif des Aiguilles Rouges, région du Mont Blanc, altitude 2300 $\mathrm{m})$. Ces stations, placées dans des sites isolés, mesurent la température de l'air, le vent et la hauteur de neige grâce à un capteur à ultrasons. Elles sont alimentées par l'énergie solaire et transmettent leurs données via satellite.
Les difficultés pour interpréter ces mesures sont de deux sortes:

- d'une part, un problème de représentativité spatiale car dans une même zone montagneuse l'influence du versant et de l'altitude sur l'épaisseur du manteau neigeux est primordiale ;

- d'autre part, l'existence dans la physique de la neige de nombreux effets de seuil (absence/présence de neige au sol, précipitations solides/liquides, changements de phase) qui font qu'il est parfois difficile de relier la hauteur de neige à un instant donné aux paramètres météorologiques classiques comme la température et les précipitations.

L'observation des hauteurs de neige moyennes décadaires, mesurées sur le site du Col de Porte (1340m, Chartreuse, figure 3 ) fin décembre et mi-février, montre bien la variabilité de ce paramètre à la fois entre hivers, et au sein d'un même hiver entre les deux décades représentées. Cette variabilité est nettement supérieure à celle observée sur les mesures de température et de précipitations. De l'observation de la série, il ressort que des séries d'hivers fortement enneigés (fin des années 60 et début des années 80) alternent avec des hivers peu enneigés (milieu des années 70 , fin des années 80) sans qu'il existe de périodicité établie ou de tendances évidentes. De plus, on peut remarquer qu'il n'est pas rare de trouver des épaisseurs de neige faibles la troisième décade de décembre. Enfin, si l'on s'intéresse aux hivers déjà mentionnés dans cet article (88-89, 89-90, 92 93), on constate qu'ils sont parmi les hivers les moins enneigés mais qu'ils peuvent être comparés aux hivers 63-64, 72-73 (pour le début de saison) et 78-79. Des études (Navarre et al., 1990) menées sur une trentaine de sites ont permis de montrer qu'on retrouvait un comportement similaire sur l'ensemble des Alpes et des Pyrénées, les périodes de fort et faible enneigement pouvant légèrement varier d'une zone à une autre.

On constate également des différences entre les Alpes du Nord et les Alpes du Sud, 1'enneigement étant en général plus faible et plus variable dans ces dernières. Certains hivers sont fortement enneigés dans les Alpes du Nord et très peu dans les Alpes du Sud, et vice versa. Ainsi, si l'on compare la hauteur de neige moyenne, la deuxième décade de février, à Courchevel (1775m, Alpes du Nord, Vanoise) et à Isola (1910m, 


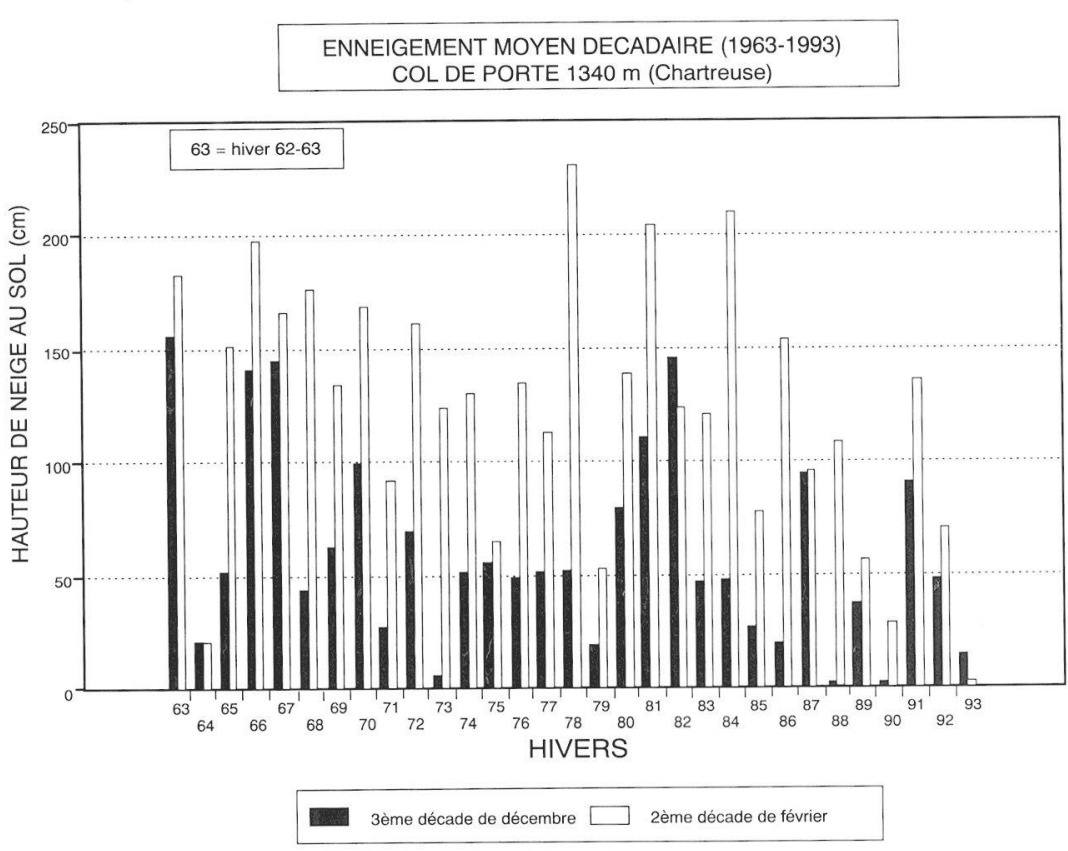

Figure 3 - Hauteur moyenne de neige $(\mathrm{en} \mathrm{cm}$ ), la troisième décade de décembre et la deuxième décade de février, au laboratoire du col de Porte, Chartreuse, $1340 \mathrm{~m}$
Alpes du Sud, Mercantour), on constate qu'elles sont très faiblement corrélées, car ces sites appartiennent à des zones climatiques très différentes : un enneigement supérieur à la moyenne dans une station ne correspond pas forcément à un enneigement supérieur à la moyenne dans l'autre (figure 4). Ce n'est évidemment pas le cas si l'on compare Courchevel à Flaine, située également dans les Alpes du Nord (1640m, Chablais, figure 5, la carte des massifs étant présentée en figure 7).

Il est donc clair que l'enneigement a une forte variabilité, à la fois spatiale et temporelle. Cela pose évidemment de gros problèmes pour le tourisme hivernal, qui depuis 20 ou 25 ans, a augmenté simultanément son poids économique dans les régions de montagne et sa dépendance vis-àvis de la neige. Mais si l'on remarque aujourd'hui que certains hivers récents sont très faiblement enneigés, on avait omis de constater le caractère exceptionnellement enneigé des hivers de la fin des années 60 ou du début des années 80 .

Enfin, il ne faut pas oublier qu'un hiver peut être classé peu ou beaucoup enneigé selon le critère choisi. Ainsi, 1'hiver 92-93, faiblement enneigé à moyenne et basse altitude, l'a été beaucoup plus à haute altitude, car les quantités de précipitations extrêmement importantes observées de mi-octobre à mi-décembre 92 y sont tombées sous forme de neige.

L'APPORT DE LA MODÉLISATION NUMÉRIQUE
Comment relier les principales caractéristiques de l'enneigement aux variables météorologiques classiques comme la température, les précipitations,...? L'épaisseur de neige un jour donné n'est pas liée aux conditions météorologiques de ce jourlà, mais est la conséquence de l'ensemble des processus physiques qui ont permis à la neige de se déposer, de se tasser, de fondre en partie, de regeler...

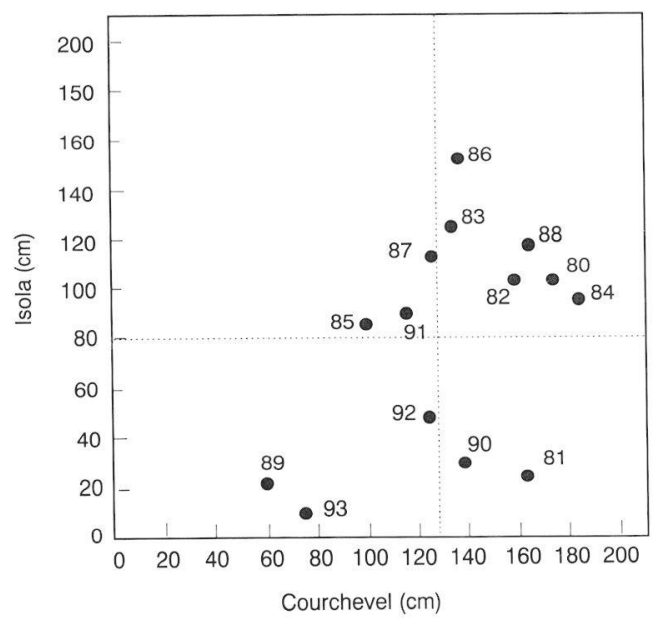

Figure 4 - Hauteur moyenne de neige $(\mathrm{en} \mathrm{cm})$, la deuxième décade de février à Isola (Mercantour, 1920 m) en fonction de la hauteur moyenne de neige à Courchevel (Vanoise, 1775 m) pendant la même décade, de 1980 à 1993 (les pointillés correspondent aux valeurs moyennes sur toute la période)

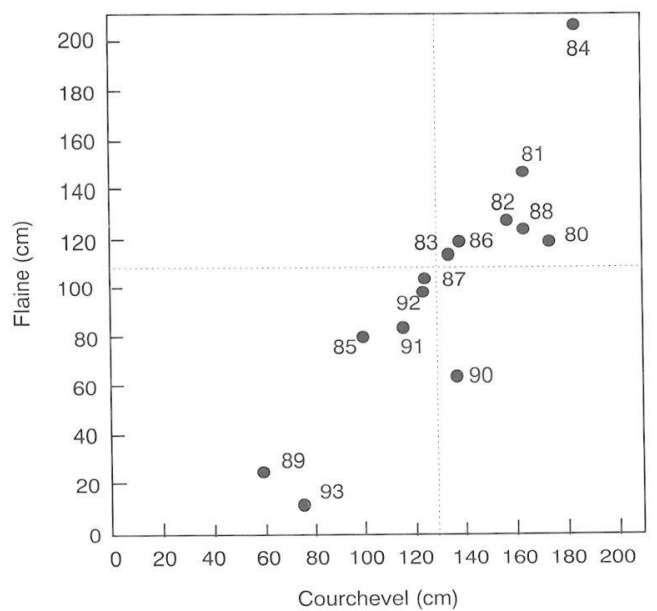

Figure 5 - Hauteur moyenne de neige (en cm) la deuxième décade de février à Flaine (Chablais, 1640 m) en fonction de la hauteur moyenne de neige à Courchevel pendant la même décade de 1980 à 1993 (les pointillés correspondent auxvaleurs moyennes sur toute la période) 
Le modèle Crocus

C'est pour cela qu'on a recours à des modèles numériques comme le modèle Crocus (Brun et al., 1989, 1992). Crocus est un modèle unidimensionnel d'évolution du manteau neigeux qui, à partir d'un état initial et de la connaissance des flux et des variables météorologiques au-dessus de la surface au pas de temps horaire, calcule l'évolution interne du manteau neigeux. Les paramètres d'entrée sont la température et l'humidité de l'air, la force du vent, les rayonnements solaire et infrarouge incidents, ainsi que les quantités et les phases des précipitations.

Le manteau neigeux est divisé en couches parallèles au sol et les variables sont les profils de température, de densité, de teneur en eau liquide, ainsi que le type de grains de neige à chaque niveau (figure 6). Les phénomènes physiques pris en compte par le modèle sont :

- les échanges d'énergie à l'intérieur du manteau neigeux et aux interfaces avec le sol et l'atmosphère,

- l'absorption du rayonnement solaire en fonction de la profondeur,

- les échanges de masse dus aux précipitations et à la fonte,

- la percolation de l'eau liquide,

- le tassement,

- la métamorphose de la neige.

L'albédo, qui dépend fortement du type de neige en surface, est également calculé.

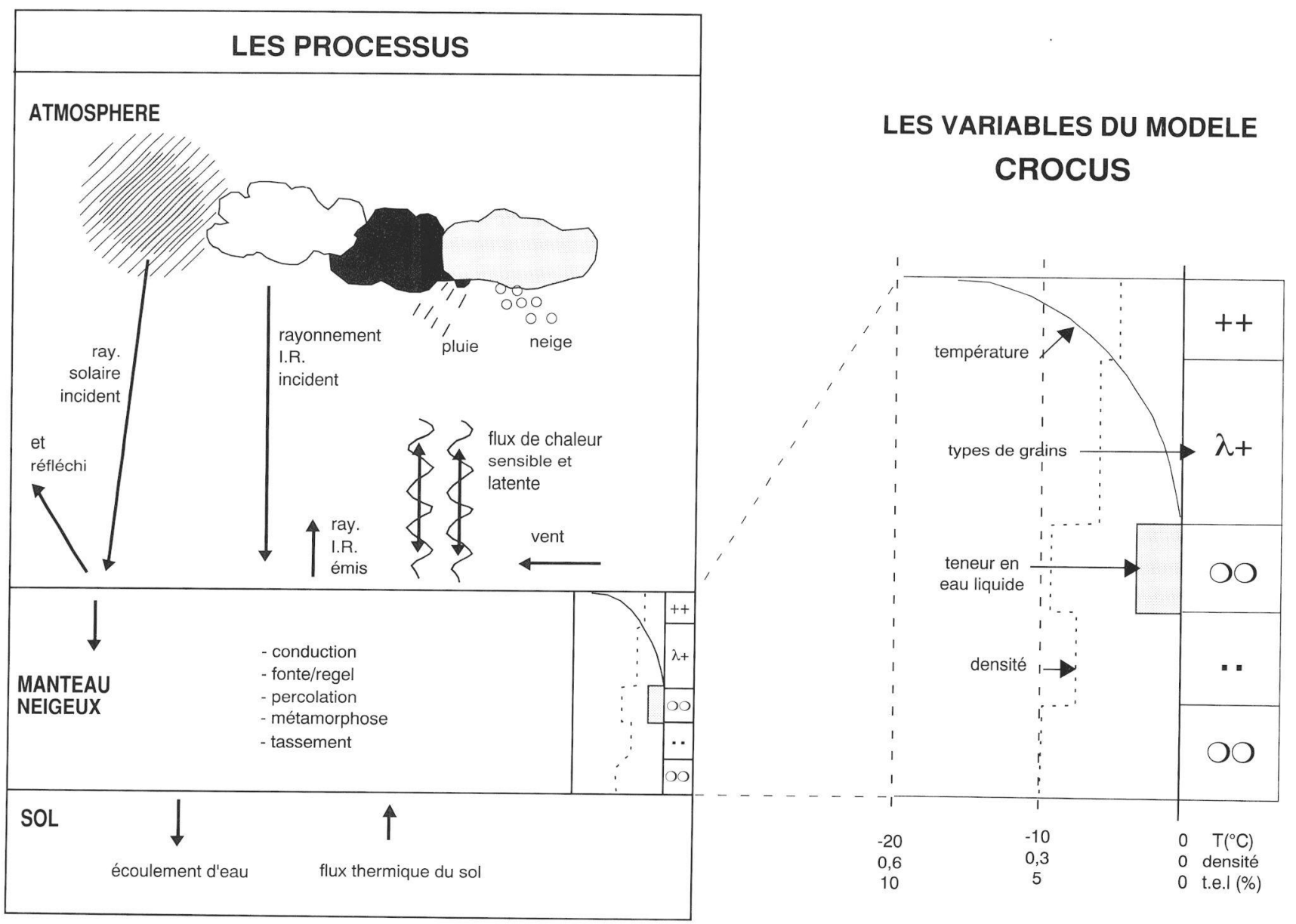

Figure 6 - Schéma des principaux processus contrôlant l'évolution du manteau neigeux

Le modèle Safran

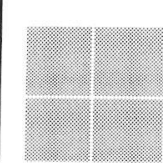

Le modèle Crocus a été testé et validé sur un site instrumenté, mais, pour l'utiliser à l'échelle régionale, un système automatique d'analyse météorologique appelé Safran a été développé (Durand et al., 1994). L'échelle retenue est celle du massif (figure 7), soit environ $1000 \mathrm{~km}^{2}$, déjà utilisée par Météo-France pour la prévision des risques d'avalanches. Ce système fournit les données d'entrée de Crocus pour chaque massif sur différents versants, avec une résolution verticale de 


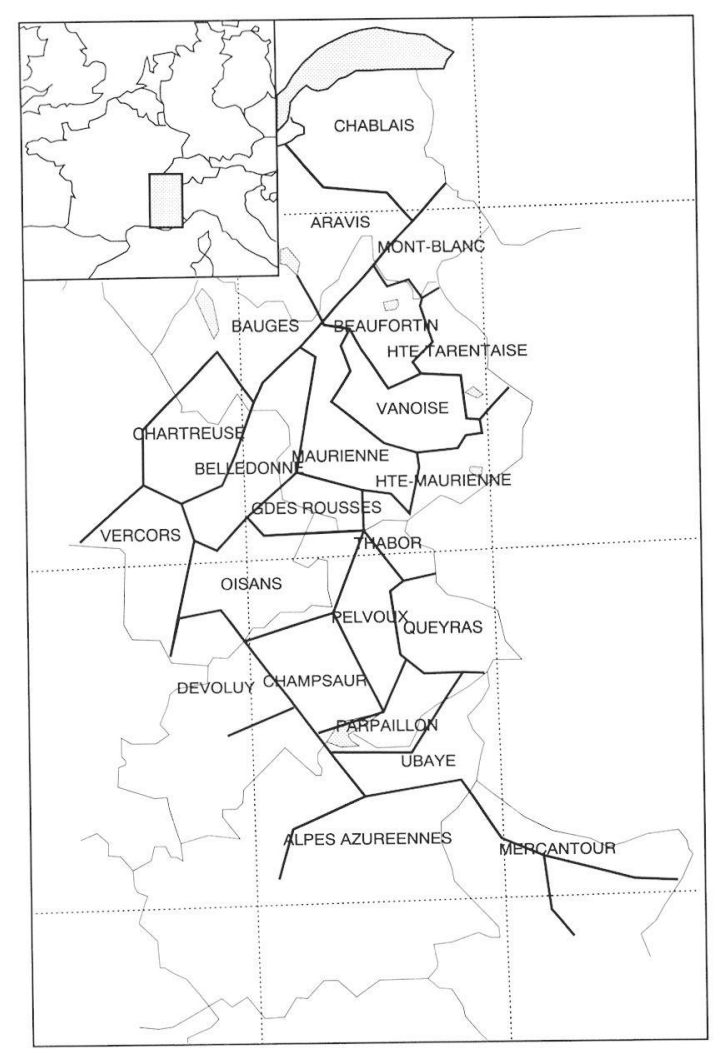

$300 \mathrm{~m}$ pour permettre la simulation de manteaux neigeux «typiques» pour chaque zone. Pour cela, il utilise deux types de données:

- des sorties de modèles météorologiques (Péridot ou CEPMMT) ;

- toutes les observations classiques au sol ou en altitude (l'introduction de données satellitaires et radar est en cours de test).

Le système utilise un schéma de type «interpolation optimale» pour estimer les principales variables (température, vent, humidité, nébulosité). Il s'agit d'une méthode largement utilisée pour le problème de l'assimilation des données en météorologie : l'analyse est faite à partir d'une première ébauche (constituée ici par les résultats des modèles météorologiques) corrigée dans un deuxième temps grâce aux observations. Les termes de rayonnement sont calculés grâce à un modèle de transfert radiatif (Ritter et Geleyn, 1992). Les quantités de précipitations sont estimées à partir de champs climatologiques calculés avec la méthode Aurelhy (Bénichou et Le Breton, 1987) et d'observations.

Figure 7 - Carte des massifs utilisés par Safran pour l'analyse des paramètres météorologiques

\section{La chaîne Safran-} Crocus et la simulation de l'enneigement

(A

Cette chaîne Safran-Crocus a été développée pour servir d'aide au prévisionniste du risque d'avalanches. Pour cela, elle est couplée au système expert Mepra qui estime les risques d'avalanches à partir de l'état du manteau neigeux simulé par Crocus. Elle a notamment été utilisée lors des Jeux Olympiques d'hiver d'Albertville en 1992. Pour valider son apport en ce qui concerne le suivi de l'enneigement sur de longues périodes, une simulation de l'épaisseur de neige sur 37 sites de test (répartis sur la plupart des massifs de 900 à 3000 m) a été réalisée sur une période de 11 ans (de l'été 1980 à l'été 1991). Pour cela, chacun des sites sélectionnés est caractérisé par son massif d'appartenance, son altitude et son exposition.

Safran estime alors pour chacun des sites déjà caractérisés les paramètres météorologiques, puis Crocus est mis en œuvre sur la période complète, sans aucune réinitialisation ni rappel à la réalité. La comparaison quotidienne des hauteurs de neige mesurées et simulées est très satisfaisante pour la plupart des postes (un exemple est donné en figure 8), ce qui assure d'une bonne représentation de la climatologie nivale. Seules les mesures de quelques sites sont mal restituées : les limites de cette approche sont principalement l'absence de prise en compte du transport de neige par le vent et des phénomènes d'échelle inférieure au massif (sites à climatologie particulière, phénomènes d'inversion dans les vallées encaissées). Il faut donc bien garder à l'esprit que les simulations réalisées avec un tel outil ne peuvent représenter l'extrême variabilité du manteau neigeux à petite échelle. Néanmoins, elles donnent entière satisfaction pour les sites ouverts, raisonnablement éloignés des lignes de crêtes où les effets locaux dus au vent sont prépondérants.

Figure 8 - Comparaison de l'enneigement simulé et mesuré pendant 10 hivers à La Clusaz (Aravis, $1500 \mathrm{~m}$ )

\section{Apport pour l'étude de la climatologie nivale}

Une des applications possibles de ces outils est l'étude de la climatologie nivale à l'échelle des Alpes françaises. En effet, le principal intérêt de l'utilisation de Safran et Crocus dans ce domaine est la possibilité d'obtenir des mesures comparables (en altitude, exposition) d'un massif à un autre, ce qui n'est pas possible avec les mesures brutes. De plus, on a facilement accès à des paramètres mesurés dans très peu d'endroits, comme la durée de l'enneigement. Ainsi, à partir des résultats d'une simulation de 11 ans, faite cette fois-ci pour tous les massifs, avec un 


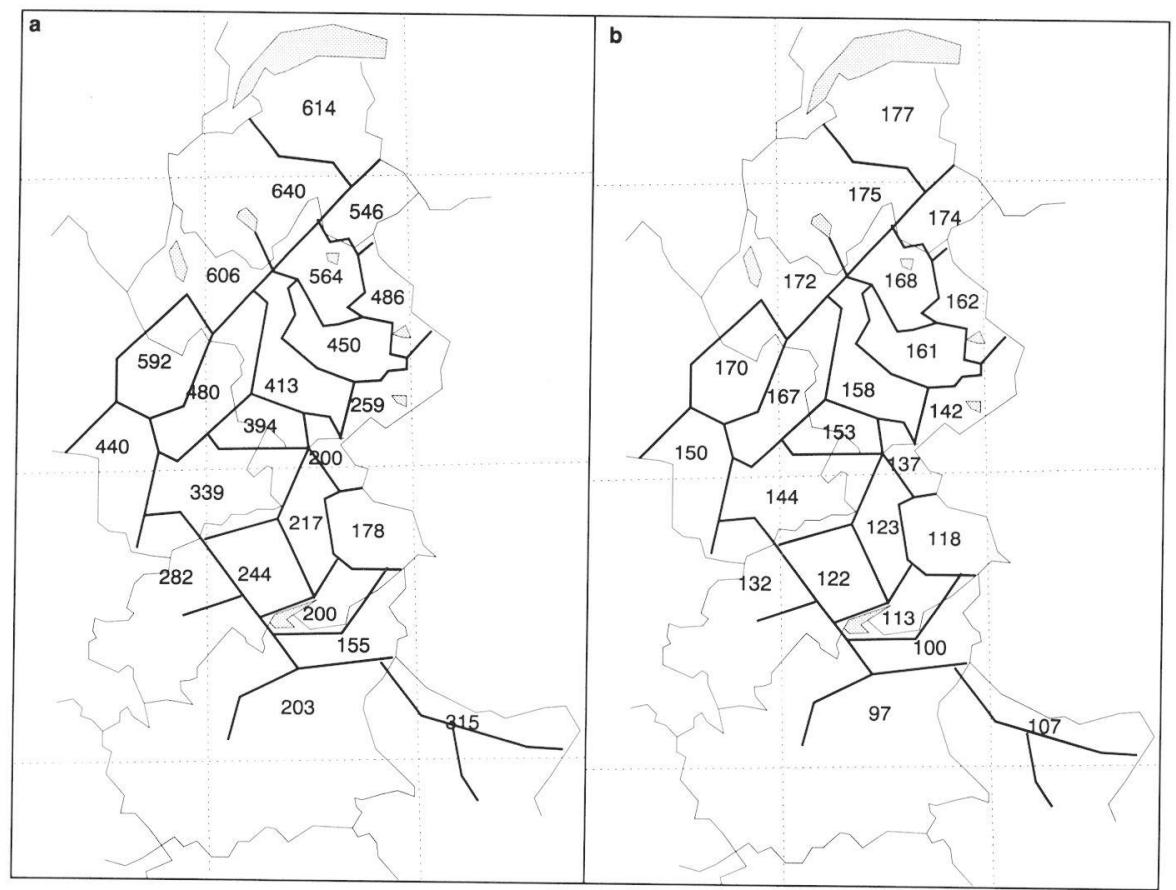

Figure 9 - a : quantité moyenne annuelle de précipitations tombées sous forme de neige à $1500 \mathrm{~m}$ sur la période 80-91 analysées par Safran (en équivalent en eau, en mm par an). b : durée moyenne annuelle de l'enneigement (en jours par an) à la même altitude et pour la même période simulée par Crocus.

pas régulier de $300 \mathrm{~m}$ en altitude et pour une surface horizontale, on peut obtenir des éléments intéressants à l'échelle des Alpes françaises. Par exemple, à 1500m (figure 9 a), on constate que les quantités moyennes annuelles de précipitations sous forme de neige, analysées par Safran, sont maximales au Nord-Ouest, sur les Préalpes du Nord. Elles décroissent ensuite progressivement en allant vers le Sud-Est. On passe de 640 à moins de $200 \mathrm{~mm}$ par an (en équivalent en lame d'eau). Le massif du Mercantour, à l'extrême Sud, constitue une exception puisqu'il reçoit en moyenne $315 \mathrm{~mm}$ par an sous forme de neige.

Cette répartition est conditionnée par les principaux types de temps qui affectent les Alpes. Ainsi, les régimes de nord-ouest apportent des quantités de neige importantes sur les Alpes du Nord et pratiquement rien sur les Alpes du Sud. Alors que les régimes de sud-est, associés à une dépression dans le Golfe de Gênes, donnent des quantités de précipitations importantes localisées sur l'extrême Sud-Est. Cette grande variabilité (rapport de 1 à 4) se retrouve aussi sur la durée moyenne annuelle de l'enneigement, puisqu'on a des différences de plus de deux mois entre le Nord et le Sud (figure 9b).

LE DEVENIR DE L'ENNEIGEMENT

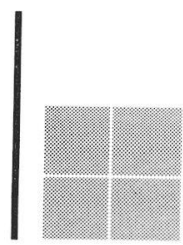

\section{Sensibilité a priori}

Grâce à l'examen de séries ponctuelles de mesures et à la modélisation, il est aujourd'hui possible d'avoir une bonne connaissance des caractéristiques de l'enneigement actuel des Alpes françaises.

Pour ce qui concerne le devenir de l'enneigement dans le cadre d'un possible réchauffement global, et vu la complexité de la physique du manteau neigeux, on peut s'attendre à ce que sa sensibilité varie selon le massif, l'altitude ou le paramètre météorologique perturbé. En l'absence d'un scénario fiable d'évolution du climat à l'échelle des Alpes, il est préférable d'étudier en détail la sensibilité du manteau neigeux, que ce soit à partir de la connaissance que nous avons de son état actuel, ou grâce à la modélisation.

La neige a quelques atouts dans son jeu pour résister à un réchauffement du climat. En effet, elle se comporte comme un réservoir de froid qui crée des conditions favorables à son maintien : son albédo fort et sa forte émissivité infrarouge lui permettent de limiter au maximum l'énergie radiative absorbée en surface. De plus elle contribue à refroidir les basses couches de l'atmosphère. Certains glaciers dans le monde survivent uniquement grâce à ce phénomène. 


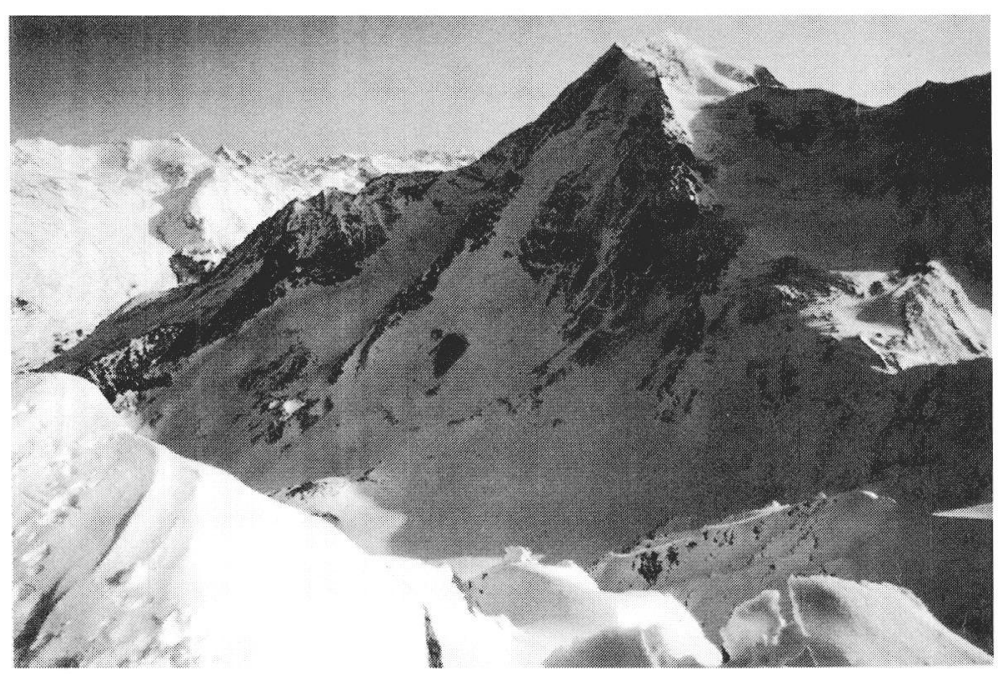

Figure 10 - La neige dans les Alpes (photo Patrick Le Floch)
En présence d'un réchauffement atmosphérique, un manteau neigeux à température négative pourra, comme un sol classique, évacuer l'énergie incidente supplémentaire en augmentant son émission infrarouge, et donc, sa température de surface. Par contre, lorsque le point de fusion est atteint en surface, ce mécanisme n'est plus possible ; une portion de l'énergie incidente supplémentaire va être utilisée pour faire fondre une partie du manteau neigeux. Tant que la quantité d'eau liquide présente à l'intérieur du manteau neigeux reste limitée près de la surface, tout refroidissement atmosphérique permettra un regel rapide et le manteau neigeux pourra facilement reconstituer sa réserve en froid. Mais, lorsque la teneur en eau liquide devient importante, la percolation de l'eau dans le manteau neigeux débute et ce dernier perd de la masse. En outre, cette présence d'eau liquide diminue fortement les chances de regel et de refroidissement de la neige. A ce stade, le manteau neigeux est fortement et durablement sensible à tout réchauffement de l'atmosphère.

Par conséquent, tant que l'on est en présence de conditions froides (cas de l'hiver pour les altitudes élevées), l'influence d'un réchauffement sera très faible sur la quantité de neige au sol. Mais par contre, il faut s'attendre à ce que l'enneigement soit particulièrement sensible lors des périodes de fonte (où l'eau liquide est présente dans tout le manteau neigeux). Ces périodes sont limitées au printemps et à l'été à haute altitude, mais peuvent arriver pendant tout l'hiver à moyenne altitude, associées à des périodes de redoux.

Sensibilité à la variation de certains paramètres météorologiques

Grâce à la modélisation numérique, il est possible d'effectuer de nombreux tests de sensibilité. La démarche adoptée ici consiste à modifier les données météorologiques d'entrée de Crocus, et à comparer les résultats à ceux de la simulation correspondant aux conditions actuelles. Mais la nature ne change pas un paramètre à la fois et lorsqu'on cherche à estimer l'impact d'un scénario climatique particulier sur l'enneigement dans les Alpes, comme celui correspondant à un doublement du gaz carbonique $\left(\mathrm{CO}_{2}\right)$ dans l'atmosphère, le problème devient plus délicat. Le problème a été résolu en deux étapes. Dans un premier temps, les variations des principaux paramètres météorologiques sur la région des Alpes ont été tirées de l'étude des résultats de deux simulations du modèle de circulation générale Emeraude (Mahfouf et al., 1992) correspondant au climat actuel et à un scénario «doublement du $\mathrm{CO}_{2}$ ». Ensuite, ces mêmes variations ont été utilisées pour modifier les données météorologiques (fournies par Safran) qui ont déjà servi au calcul de la climatologie nivale par Crocus. Bien sûr, ce type d'approche est loin d'être satisfaisant vu la dimension de la maille des modèles de circulation générale (et donc leur mauvaise représentation du relief), mais l'essentiel est d'obtenir des perturbations cohérentes entre elles des différents paramètres.

Les perturbations des variables météorologiques correspondant au scénario «doublement du $\mathrm{CO}_{2}$ » ont été les suivantes :

- rayonnement solaire direct : $+10 \%$

- rayonnement solaire diffus incident : $-10 \%$

- rayonnement infrarouge incident : $+3 \%$

- température de l'air : $+1,8^{\circ} \mathrm{C}$

- transformation de la neige en pluie lorsque la nouvelle température de l'air dépasse $1,5^{\circ} \mathrm{C}$.

Sans entrer dans le détail des résultats, on peut dire que deux paramètres (ou groupes de paramètres) sont importants pour le manteau neigeux. Le premier est la température de l'air (et le rayonnement infrarouge, qui lui est lié). Les tests effectués confirment le raisonnement tenu dans le paragraphe précédent. Une limite se dessine entre 2000 et $2500 \mathrm{~m}$. Au-dessus, l'effet d'une augmentation de la température, homogène suivant le massif, est faible et limité au printemps. Au-dessous, par contre, l'effet est de plus en plus important au fur et à mesure que l'on descend en altitude et que l'on va vers le Sud. 


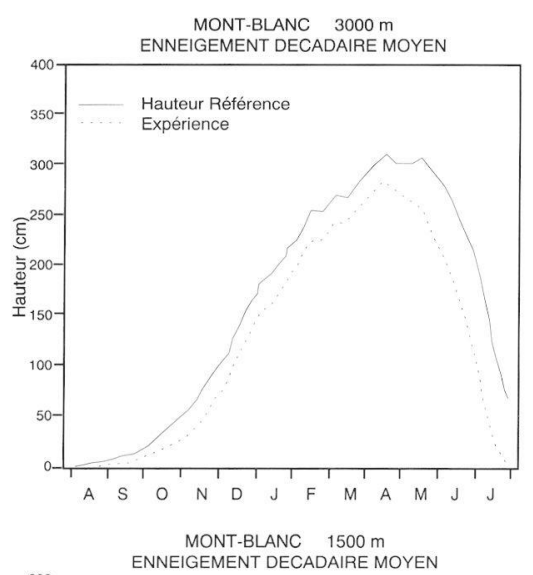

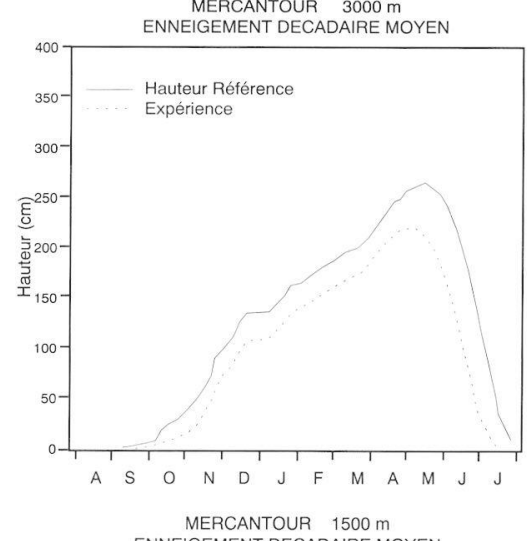

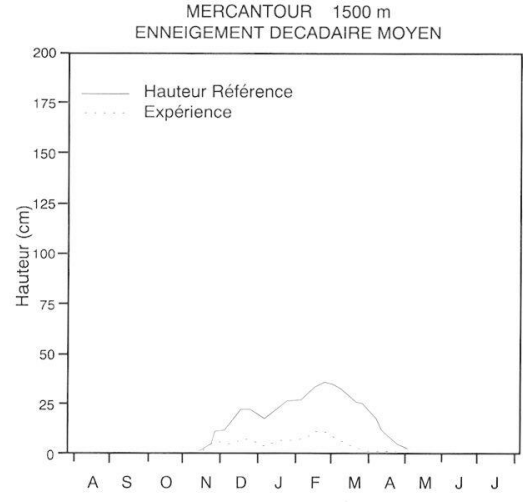

Figure 11 - Enneigement décadaire moyen simulé par Safran et Crocus pour les massifs du Mont-Blanc (Alpes du Nord) et du Mercantour (Alpes du Sud) à 3000 et $1500 \mathrm{~m}$ d'altitude pour les conditions 1980-91 (traits continus) et dans le cas de l'expérience de doublement du $\mathrm{CO}_{2}$ (traits pointillés)
Le deuxième paramètre important est constitué par l'évolution des quantités de précipitations. Son effet est a priori plus facile à appréhender. Par exemple, si l'on augmente les quantités de précipitations, les calculs montrent qu'on retrouve à peu près la même variation sur l'épaisseur de neige. Par contre la variation est plus faible pour ce qui concerne la durée de l'enneigement. En effet, la neige tombée en surplus disparaît très rapidement parce qu'elle fond en fin de saison, au moment où la température est élevée. Le résultat est cohérent avec les données de la figure 8 , où l'on voit que la variabilité très importante des quantités de précipitations se retrouve nettement atténuée sur les durées d'enneigement. On obtient des résultats symétriques si l'on diminue les quantités de précipitations.

L'impact du scénario climatique décrit ci-dessus est très important, que ce soit pour l'épaisseur de neige ou la durée de l'enneigement. Cette dernière, à $1500 \mathrm{~m}$, diminue de 30 à 40 jours par an en moyenne, passant par exemple de 174 à 132 jours par an dans la région du MontBlanc et de 107 à 59 jours par an dans le Mercantour. Pour ce qui concerne l'épaisseur de neige (figure 11), les régions des Alpes du Nord sont plus touchées si on regarde les valeurs absolues, tandis que les Alpes du Sud le sont plus si on examine la perte en pourcentage.

La figure 12 décrit l'évolution de la hauteur moyenne de neige,

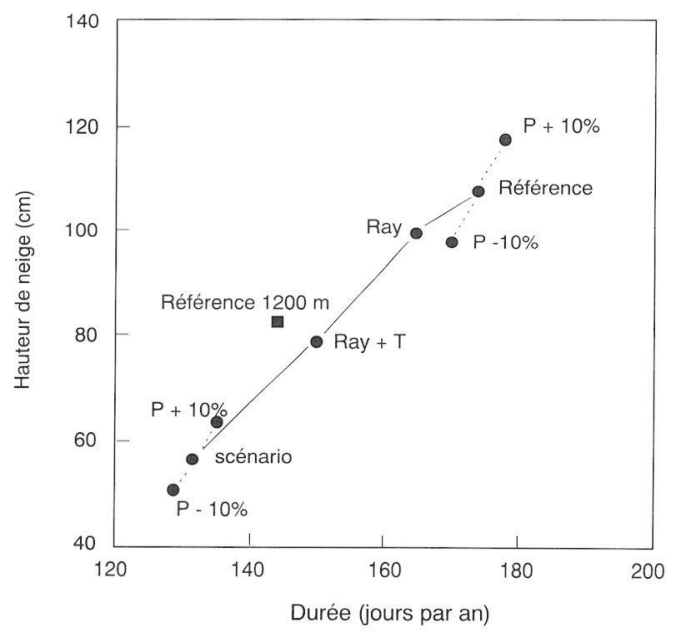

Figure 12 - Hauteur de neige moyenne la première décade de mars en fonction de la durée moyenne de l'enneigement dans le Mont-Blanc à 1500m simulées par Crocus dans la simulation de référence et pour diverses expériences de sensibilité :

Référence : simulation correspondant aux conditions 1980-91;

Scénario : résultat correspondant au scénario doublement du $\mathrm{CO}_{2}$;

Ray : modification des termes de rayonnement uniquement ;

Ray+T : modification des termes de rayonnement et de la température :

$\mathrm{P}+10,(\mathrm{P}-10)$ : augmentation (diminution) des quantités de précipitations de $10 \%$ à partir de l'état de référence ou du scénario ;

Référence $1200 \mathrm{~m}$ : point de référence pour la simulation à $1200 \mathrm{~m}$. pendant la première décade de mars, en fonction de la durée moyenne de l'enneigement telles qu'elles ont été simulées par Crocus pour le massif du Mont-Blanc, à $1500 \mathrm{~m}$, dans la simulation de référence et pour diverses expériences de sensibilité. On remarque bien les effets différenciés de la température et des précipitations. En particulier, il semble improbable qu'une augmentation des précipitations puisse compenser l'effet du réchauffement.

Alors que l'on vient d'utiliser des outils sophistiqués pour estimer l'impact d'un réchauffement global sur l'enneigement, il est légitime de se demander si, avec des méthodes plus simples on aurait obtenu le même résultat. Le gradient vertical moyen de température dans l'atmosphère étant de l'ordre de $-0,6^{\circ} \mathrm{C}$ pour $100 \mathrm{~m}$, on pourrait s'attendre, pour un réchauffement de $1,8^{\circ} \mathrm{C}$, à retrouver à une altitude donnée des conditions similaires à celles qui existent $300 \mathrm{~m}$ plus bas actuellement. Cette hypothèse simple, si elle est satisfaisante à haute altitude, est mise en défaut en dessous de $2000 \mathrm{~m}$ sur toutes les Alpes (figure 12 pour le Mont-Blanc à $1500 \mathrm{~m}$ ). Ceci est encore une conséquence des nombreux effets de seuil qui caractérisent la physique de la neige et rendent inutilisable la méthode consistant à décaler les tranches d'altitude. Ainsi, pour trouver des caractéristiques équivalentes à celles du scénario dans la simulation de référence, il faut parfois descendre de 400 à 600 mètres ! 
Tous les moyens d'investigation utilisés ici (séries de mesures et modélisation) mettent en évidence la forte dépendance de l'enneigement vis-à-vis des conditions météorologiques, singulièrement en moyenne montagne. Cette dépendance est visible sur un site particulier, où la variabilité temporelle est très forte, mais aussi à l'échelle des Alpes françaises, où l'enneigement est conditionné par la fréquence des types de temps affectant la région. A cause de ces grosses variations, il sera très difficile de détecter un éventuel changement du climat, d'autant plus que dans les séries dont on dispose, les années exceptionnelles se présentent souvent par petites séries.

Les tests de sensibilité ont mis en évidence deux zones principales :

- d'une part les altitudes élevées (au-dessus de 2000 à $2500 \mathrm{~m}$ ) où les conditions sont assez homogènes sur l'ensemble des Alpes françaises ; les conséquences d'un réchauffement s'y feraient sentir uniquement pendant la période de fonte qui serait plus rapide et débuterait plus tôt;

- d'autre part, les altitudes moyennes et basses, où la sensibilité augmente au fur et à mesure que l'altitude diminue et que l'on se rapproche du Sud. L'enneigement y serait fortement perturbé en cas de réchauffement.

En fait, cette répartition est essentiellement due à la variabilité du manteau neigeux, et en particulier à celle de son état interne à l'échelle considérée car les perturbations sont identiques pour tous les massifs et toutes les altitudes. Même si grâce à la modélisation on peut facilement calculer l'impact de variations quelconques, il faut bien garder à l'esprit que les scénarios utilisés ici restent grossiers, car ils ne tiennent pas compte d'une possible rétroaction sol-atmosphère, et qu'ils sont tirés de modèles de circulation générale qui ne peuvent pas encore produire de scénarios réalistes à l'échelle des Alpes. De plus, les perturbations introduites ne dépendent ni du temps, ni de l'altitude, ni du massif, alors qu'un des phénomènes importants pourrait être un changement dans la fréquence des types de temps qui affectent les Alpes et qui, on l'a vu, conditionnent fortement la répartition des chutes de neige. La démarche utilisée ici, qui consiste à introduire des perturbations simples à un état initial bien représenté, est donc limitée. Néanmoins, la très forte sensibilité de l'enneigement à toute variation des paramètres météorologiques montre bien l'intérêt des recherches menées en vue d'obtenir des scénarios régionaux fiables d'évolution du climat.

Bénichou P. et O. Le Breton, 1987 : Prise en compte de la topographie pour la cartographie des champs pluviométriques statistiques. La Météorologie , $7^{\circledR}$ série, 19, 23-34.

Brun E., E. Martin, V. Simon, C. Gendre et C. Coléou, 1989 : An energy and mass model of snow cover for operational avalanche forecasting. J. of Glaciol., 121, 333-342.

Brun E., P. David, M. Sudul et G. Brunot, 1992 : A numerical model to simulate snow-cover stratigraphy for operational avalanche forecasting. J. of Glaciol., 128, 13-22.

Durand Y., E. Brun, L. Mérindol, G. Guyomarc'h, B. Lesaffre et E. Martin, 1994 : A meteorological estimation of relevant parameters for snow models. Annals of Glaciol., Vol 18 , sous presse.

Mahfouf J.-F., J.-F. Royer et D. Cariolle, 1992: A $2 *$ CO2 experiment with prescribed changes in sea surface temperature. Proceedings of the Central Symposium of the «International Space Year» Conference, Munich, Germany (ESA SP-341, July 1992), 151-155.

Navarre J.-P., J. Villecrose, E. Pahaut et J. König-Barde, 1990 : Caractères exceptionnels de l'enneigement au début de l'hiver 1989-90. La Météorologie, $7^{\mathrm{C}}$ série, 34, 40-48.

Ritter B. et J.-F. Geleyn, 1992 : A comprehensive radiation scheme for numerical weather prediction models with potential applications in climate simulations. Monthly weather review, 120, 2, 303-325. 